\title{
Alvarado versus Raja Isteri Pengiran Anak Saleha Appendicitis Scores for Diagnosis Acute Appendicitis in Children
}

\author{
TÜLIN ÖZTAŞ ${ }^{1}$ and Muhammet Asena ${ }^{1}$ \\ ${ }^{1}$ Affiliation not available
}

November 10, 2020

\begin{abstract}
Objective Although acute appendicitis is the most common emergency surgical pathology it is not easy to diagnose in pediatric patients. This study aims to compare the accuracy of Raja Isteri Pengiran Anak Saleha Appendicitis (RIPASA) and Alvarado scores in the diagnosis of acute appendicitis in children. Methodology Demographic data, examination findings, leukocyte count, neutrophil percentage, urine analysis and radiology reports of 163 consecutive patients who were underwent surgery for acute appendicitis were recorded. RIPASA and Alvarado scores of patients were calculated prospectively. Cutoff value was $>7.5$ for RIPASA score , [?]7 for Alvarado score. The accuracy of the diagnosis was confirmed by the histopathology result. The accuracy, sensitivity, specificity, positive predictive value, negative predictive value, area under the ROC curve of the RIPASA vs. Alvarado scores were evaluated. Results Negative appendectomy rate was $11.1 \%$. The accuracy rate was $76.1 \%$ in the RIPASA score vs. $68.7 \%$ in the Alvarado score. The sensitivity and specificity values were $77.9 \%, 61.1 \%$ for RIPASA score vs. $68.2 \%, 72.2 \%$ for Alvarado score, respectively. Positive predictive value was $94.1 \%$ and negative predictive value was $25.5 \%$ for RIPASA score vs. $95.1 \%, 22.1 \%$, respectively for Alvarado score. The area under the ROC curve was 0.74 for RIPASA score vs. 0.68 for Alvarado score, with no statistically significant difference between the two scores ( $\mathrm{p}>0.05$ ). Conclusions RIPASA and Alvarado scores are not superior to each other in the diagnosis of acute appendicitis in children. Clinical examination, laboratory and radiology were more valuable in the diagnosis of acute appendicitis in children.
\end{abstract}

\section{Alvarado versus Raja Isteri Pengiran Anak Saleha Appendicitis Scores for Diagnosis Acute Appendicitis in Children}

\section{Objective}

Although acute appendicitis is the most common emergency surgical pathology it is not easy to diagnose in pediatric patients. This study aims to compare the accuracy of Raja Isteri Pengiran Anak Saleha Appendicitis ( RIPASA) and Alvarado scores in the diagnosis of acute appendicitis in children.

\section{Methodology}

Demographic data, examination findings, leukocyte count, neutrophil percentage, urine analysis and radiology reports of 163 consecutive patients who were underwent surgery for acute appendicitis were recorded. RIPASA and Alvarado scores of patients were calculated prospectively. Cutoff value was $>7.5$ for RIPASA score, [?]7 for Alvarado score. The accuracy of the diagnosis was confirmed by the histopathology result. The accuracy, sensitivity, specificity, positive predictive value, negative predictive value, area under the ROC curve of the RIPASA vs. Alvarado scores were evaluated.

\section{Results}

Negative appendectomy rate was $11.1 \%$. The accuracy rate was $76.1 \%$ in the RIPASA score vs. $68.7 \%$ in the Alvarado score. The sensitivity and specificity values were $77.9 \%, 61.1 \%$ for RIPASA score vs. $68.2 \%$, 
$72.2 \%$ for Alvarado score, respectively. Positive predictive value was $94.1 \%$ and negative predictive value was $25.5 \%$ for RIPASA score vs. $95.1 \%, 22.1 \%$, respectively for Alvarado score. The area under the ROC curve was 0.74 for RIPASA score vs. 0.68 for Alvarado score, with no statistically significant difference between the two scores $(\mathrm{p}>0.05)$.

\section{Conclusions}

RIPASA and Alvarado scores are not superior to each other in the diagnosis of acute appendicitis in children. Clinical examination, laboratory and radiology were more valuable in the diagnosis of acute appendicitis in children.

Keywords: Acute appendicitis, Appendectomy, Alvarado score, RIPASA score.

What's already known about this topic?

Alvarado is one of the most commonly used scoring methods in pediatric patients.

The RIPASA Score is considered to be a better diagnostic scoring method in adults compared to Alvarado

What does this article add?

RIPASA score is considered to be a better diagnostic scoring method in adults compared to Alvarado but two scores are not superior to each other in the diagnosis of acute appendicitis in children.

Clinical examination, laboratory and radiology were more valuable in the diagnosis of acute appendicitis in children.

\section{Introduction}

Acute appendicitis is the most common emergency surgical pathology at all age groups. It is not easy to diagnose especially in children younger than 5 -years of age which can lead to an increased rate of perforation. ${ }^{1}$ Diagnosis is usually established with history, physical examination and not specific to acute appendicitis laboratory tests such as white blood count (WBC), Neutrophil percentage (NP), C-reactive protein (CRP) and imaging methods. Ultrasonography (USG) which is the first and the most preferred imaging method due to its quick practicality and non-radiation exposure. USG has disadvantages such as failure to visualize the retrocecal appendix, as well as its dependency on the performer. ${ }^{2,3}$ In patients in whom USG fails to visualize the appendix, it is recommended that the patient be monitored instead of utilizing methods such as contrast tomography (CT) or magnetic resonance imaging (MRI) or diagnostic laparoscopy. ${ }^{4}$ Complications such as abscess, perforation, peritonitis and rarely sepsis may develop during monitoring. ${ }^{5}$ Emergency operations performed in patients with suspected acute appendicitis result in a negative appendectomy with a rate of $15-27 \% \cdot{ }^{6-8}$ Many scoring systems have been developed to reduce ambiguities in the diagnosis of appendicitis. Alvarado is one of the most commonly used scoring methods in pediatric patients. ${ }^{2}$ RIPASA score, which is created by introducing some parameters such as age, gender, symptom duration, and urinalysis, which are not found in the Alvarado Score ${ }^{8,9}$ (Table 1).

The objective of this study is to compare the accuracy, sensitivity, and specificity of the RIPASA and Alvarado Scores in the diagnosis of acute appendicitis in pediatric patients.

\section{Materials and Methods}

This cross-sectional study included 163 consecutive patients who had been operated in our pediatric surgery clinic with a preliminary diagnosis of acute appendicitis between July 2018 and December 2019. During the study period, patients diagnosed with appendicitis and underwent appendectomy were included in the study. Patients younger than two years of age who had refused to cooperate in physical examination were excluded from the study.

The operation decision of the patients was made by independent surgeons based on history, examination, laboratory and radiological imaging. Patients who were scheduled for operation were evaluated by the physicians who performed the study before the operation. Patients' history was obtained, physical examination 
was performed, and age, gender, duration of symptoms, nausea, vomiting, tenderness in the right lower quadrant, migration of pain to the right lower quadrant, defensive, rebound, rovsing, fever, WBC, NP, CRP, urinalysis and radiology reports were recorded in the pre-prepared scoring form.

Serum CRP value $>5 \mathrm{mg} / \mathrm{L}, \mathrm{WBC} \mu \mathrm{L}>10,000 / \mathrm{L}, \mathrm{NP}>75 \%$ were considered high. ${ }^{10} \mathrm{USG}$ reports were evaluated in three categories: appendix not visualized, normal appendix and acute appendicitis. In US, wall thickness of the appendix $>6 \mathrm{~mm}$ was interpreted as acute appendicitis. ${ }^{11}$

RIPASA and Alvarado scores were calculated prospectively only for the study by two physicians independent of the study. The possibility of appendicitis was divided into three groups according to scoring systems. RIPASA score was considered very high at [?]12, high at 7.5-11.5, moderate at 5-7 and of low probability at $<5$, Alvarado score was considered high at [?]7, moderate at $5-6$, of low probability at $0-4 .{ }^{6,12}$

Appendix specimens were evaluated microscopically in three categories: lymphoid hyperplasia, acute appendicitis, complicated appendicitis (gangrenous appendicitis or perforated). ${ }^{10}$ The accuracy of the diagnosis was confirmed by the histopathology result.

The accuracy rate, sensitivity, specificity, negative predictive values (NPV), positive predictive values (PPV), reciever operator characteristics curve (ROC) analysis were performed for RIPASA and Alvarado scoring systems. In addition, sensitivity and false positivity, PPV of two scores were evaluated in patients with complicated appendicitis and by age.

The study was approved by the Clinical Studies Ethics Committee of Health Sciences University (06.07.2018 / No: 119).

\section{Statistical methods}

Data obtained in the study were statistically analyzed using SPSS Statistics for Windows, Version 22.0. (IBM Corp. Released 2013. Armonk, NY). Categorical variables were expressed as numbers (n) and percentages (\%) and the Kolmogorov-Smirnov test was used to examine the normal distribution of continuous data. Numerical variables with normal distribution were shown as mean \pm standard deviation. Normally distributed numerical variables were compared by Student's $T$ test. Cut off value was $>7.5$ for RIPASA score vs. [?]7 for Alvarado score. ${ }^{13}$ Pearson's Chi-Square Test was used to determine whether there is a difference between RIPASA and Alvarado scores in terms of accuracy, sensitivity, specificity, positive predictive value, negative predictive value, area under the ROC curve (AUC). In all data, $\mathrm{p}<0.05$ was considered statistically significant.

\section{Results}

Of the 163 patients included in the study, $31.9 \%$ were girls and $68.1 \%$ were boys, and the mean age was 11.2 +- 3.3 years (2-17 years). The diagnosis of appendicitis was confirmed by histopathological examination in 145 of the patients who had been operated with a preliminary diagnosis of acute appendicitis. Pathology reports demonstrated $11.1 \%$ lymphoid hyperplasia, $68.7 \%$ acute appendicitis, $20.2 \%$ complicated appendicitis (14.7\% gangrenous appendicitis, $5.5 \%$ perforated appendicitis). Of the patients, $88.3 \%$ had WBC elevation, $76.6 \%$ had NP elevation and $57.6 \%$ had CRP elevation. In $81.6 \%$ of patients, USG was reported as acute appendicitis. (Table 2). The accuracy rate of were WBC, NP, CRP and USG 87.1\%, 71.8\%, 58.4\% and $84.6 \%$, respectively. In our study, the negative appendectomy rate was $11.1 \%$ when patients are evaluated by clinical laboratory and US.

The distribution of scores was as follows. RIPASA was very high in $6.1 \%$, high in $70.5 \%$, moderate in $25.7 \%$ and low in $0.6 \%$ whereas Alvarado was high in $63.8 \%$, moderate in $25.1 \%$ and low in $11.0 \%$. The nine (5.5\%) patients whose RIPASA scores were greater than 7.5 had no appendicitis on pathohistological analysis, and $19(11.6 \%)$ patients whose scores were lower than 7.5 had appendicitis. The five (3.1\%) patients whose Alvarado scores were 7 and more had no appendicitis, and $36(22 \%)$ patients whose scores were lower than 7 had appendicitis on pathohistology (Table 3). 
RIPASA score had sensitivity of $78.5 \%$, false positivity of $2.4 \%$ and accuracy rate of $77.6 \%$ in boys vs. $76.6 \%$, $1.8 \%, 72.5 \%$, respectively in girls. It was observed that the sensitivity, false positivity and accuracy rate of the RIPASA score did not vary by gender $(\mathrm{p}=0.20)$. The sensitivity, false positivity, accuracy rate of Alvarado score was $68.1 \%, 2.4 \%, 68.4 \%$ in boys vs. $68.7 \%, 0.6 \%, 69.2 \%$ in girls, respectively. It was observed that the sensitivity, false positivity and accuracy rate of the Alvarado score did not vary by gender $(\mathrm{p}=$ $0.41)$.

In our study, sensitivity and specificity values of RIPASA score were $77.9 \%, 61.1 \%$ and sensitivity and specificity of Alvarado score were $68.2 \%, 72.2 \%$, respectively. PPV and NPV were $94.1 \%, 25.5 \%$ in RIPASA score vs. $95.1 \%, 22.0 \%$ in the Alvarado score, respectively. The accuracy rate was $76.0 \%$ in the RIPASA score vs. $68.7 \%$ in the Alvarado score. With ROC curve analysis applied, the area under the curve (AUC) was 0.74 (0.66-0.81) in the RIPASA score vs. 0.68 (0.60-0.76) in the Alvarado score. There was no statistically significant difference between RIPASA and Alvarado scores in terms of accuracy, sensitivity, specificity, PPV, NPV, AUC (p = 0.93) (Table 4).

In cases with complicated appendicitis, RIPASA score had sensitivity of $93.9 \%$, false positivity of $0 \%$, accuracy rate of $93.9 \%$ whereas Alvarado score had sensitivity of $90.9 \%$, false positivity of $0 \%$ and accuracy rate of $90.9 \%$. The sensitivity and accuracy rate of the two scores were higher in cases of complicated appendicitis than in cases of acute appendicitis. In patients with complicated appendicitis, no statistically significant difference was found between RIPASA and Alvarado scores in terms of sensitivity, false positivity, and accuracy rate $(\mathrm{p}=0.31)$ (Table 5$)$.

Since the number of foreign patients $(\mathrm{n}=5)$ was very low in our study, there was no significant change as a result of the inclusion of these patients.

\section{Discussion}

The diagnosis of acute appendicitis in pediatric patients remains a problem. It has been reported that WBC and NP increase statistically significantly in patients with acute appendicitis, and that WBC and CRP elevation may help the diagnosis. ${ }^{3,14}$ The results of our study showed that WBC, NP and CRP elevations were found in patients with appendicitis, but the accuracy rate of these tests was low. It was stated that the appendix imaging rate by US, which was performed to confirm the diagnosis, ranged between $2-82 \%{ }^{5}$ It is aimed to reduce the negative appendectomy by using scoring systems in patients with suspected acute appendicitis. Our study found that clinical laboratory and radiology were more valuable in the diagnosis of acute appendicitis in children. The accuracy rate was low in RIPASA and Alvarado scores.

Scoring systems have been reported to have more diagnostic value in men since there are fewer etiological causes causing pain in the right lower quadrant. ${ }^{14}$ Alvarado score was reported to have higher sensitivity in males and higher false positivity in females. ${ }^{2,13}$ The results of our study showed that there was no relationship between gender and the sensitivity, false positivity or accuracy rate of the RIPASA and Alvarado scores.

In the study by Shamy et al, Alvarado score had a sensitivity of $95.9 \%$, specificity of $33.3 \%$, PPV of $97 \%$, NPV of $87.8 \%$, whereas Pogorelic et al. reported these values as $76.1 \%, 72 \%, 76 \%$, and $64 \%$, respectively. 6,14 It was stated that PPV and NPV components of Alvarado score are not sufficient on their own for taking the decision for surgery. ${ }^{3,6}$ In our study, only operated patients were evaluated and the specificity (72.2\%), PPV $(95.1 \%)$ values were consistent with the literature, while the sensitivity $(68.2 \%)$ and NPV $(22.1 \%)$ was low. The results of our study suggest that this difference is due to the selected patients who had been operated for acute appendicitis.

It was reported that in patient with low Alvarado scores can be discharged while observation was recommended for patients with moderate scores and surgery for patients with high scores. ${ }^{6,7}$ The results of our study showed that the diagnostic value of the Alvarado score was low since reliable history could not be obtained in young children and that the diagnosis of appendicitis could not be ruled out in patients with low scores.

In the diagnosis of acute appendicitis, RIPASA score was reported to have a diagnostic accuracy rate of 91.8- 
97.6\%, sensitivity of $93.1-98.8 \%$, specificity of $96-63 \%$, NPV of $10.1-97.6 \%$ and PPV of $85.3-98.8 \% .{ }^{12,15-21}$ In our study, accuracy (76.1\%), sensitivity $(77.9 \%)$ and specificity $(61.1 \%)$ were lower, and PPV (94.1\%) and NPV $(25.5 \%)$ values were in line with the previous studies. The results of our study suggest that the sensitivity and specificity of RIPASA score was low for the migration of pain to the right lower quadrant in young children because the duration of the symptom was not correctly expressed. The PPV value was high because the patients who had been operated for acute appendicitis were evaluated.

In the current literature, there is no study comparing the RIPASA and Alvarado scores in the diagnosis of acute appendicitis in pediatric patients. While some studies have reported that the RIPASA score is more valuable in adult patients, ${ }^{12,15,17,18,21}$ other studies have reported higher accuracy and sensitivity but lower specificity for RIPASA score compared to Alvarado scoring system. ${ }^{16,20}$ In a study by Brientos et al, it was found that the RIPASA score had no advantage over Alvarado score. ${ }^{19}$ Another study was reported that no significant difference was found in ROC analysis between RIPASA and Alvarado scores in terms of area under curve. ${ }^{22}$ The results of our study showed that RIPASA score was not superior to Alvarado score in the diagnosis of acute appendicitis and the diagnostic values of the two scores are not high enough to affect the decision for surgery in children. In the study by Chong et al., in patients with low RIPASA score, monitoring and score repetition after 1-2 hours has been recommended and if the score decreases, the patients are discharged. On the other hand, in patients with moderate score, monitoring and score repetition or USG after 1-2 hours has been recommended. In patients with high probability, score repetition after 1 hour and if the score is still high, surgical preparation has been recommended. ${ }^{12}$ The conclusions of our study demonstrated that appendicitis could be ruled out in patients with low probability by the RIPASA scoring system whereas surgery is required in patients with very high probability. It was observed that USG could be useful in the management of patients with moderate to high probability.

Alvarado scores systems had a better diagnostic value for complicated appendicitis compared to acute appendicitis. ${ }^{11}$ The conclusions of our study Alvarado and RIPASA Scores had a higher rate of accuracy in complicated appendicitis but the two scores were not superior to each other.

The limitations of this study: this is a study from one center, multicentric study should be performed to validate this data. In this study, only patients who had been operated for acute appendicitis were evaluated. There is a need for studies to compare RIPASA and Alvarado scores in all children with abdominal pain, diagnosed and not diagnosed with appendicitis.

\section{Conclusion}

RIPASA score is considered to be a better diagnostic scoring method in adults compared to Alvarado but two scores are not superior to each other in the diagnosis of acute appendicitis in children. High scores of RIPASA and Alvarado do not have sufficient accuracy, sensitivity and specificity values on their own for establishing the diagnosis for acute appendicitis. Clinical examination, laboratory and radiology were more valuable in the diagnosis of acute appendicitis in children.

\section{References}

1 Pogorelic' Z, Josipa Domjanovic' J, Jukic' M, PericVic' TP. Acute appendicitis in children younger than five years of age: diagnostic challenge for pediatric surgeons. Surg Infect (Larchmt). 2020; 21: 239-245.

2 Mandeville K, Pottker T, Bulloch B, Liu J. Using appendicitis scores in the pediatric ed. Am Jof Emerg Med 2011; 29: 972-977.

3 Abou MB, Khalil M, Daoud N. Comparison of Alvarado score evaluation and clinical judgment in acute appendicitis. Med Arh 2014;68: 10-13.

4 van Amstel P, Gorter RR, van der Lee JH, Cense HA, Bakx R, Heji HA. Ruling out appendicitis in children: can we use clinical prediction rules? Journal of Gastrointestinal Surgery 2019; $23: 2027-2048$

5 Damburaci N, Sevinc B, Guner M, Karahan O. Comparison of Raja Isteri Pengiran Anak Saleha Appendicitis and modified Alvarado scoring systems in the diagnosis of acute appendicitis. ANZ J Surg 2020; 90 : 
$521-524$.

6 Pogorelić Z, Rak S, Mrklić I, Jurić I. Prospective validation of Alvarado score and pediatric appendicitis score for the diagnosis of acute appendicitis in children. Pediatr Emerg Care 2015; 31 : 164-168.

7 Douglas CD, Macpherson NE, Davidson PM, Gani JS. Randomised controlled trial of ultrasonography in diagnosis of acute appendicitis, incorporating the Alvarado score. BMJ 2000; 321 : 919-922.

8 Malik MU, Connelly TM, Awan F, et al. The RIPASA score is sensitive and specific for the diagnosis of acute appendicitis in a western population. Int J Colorectal Dis 2016; 32 : 491-497.

9 Siddiqui ZR, Khaliq T, Shah SA. A new simple scoring system for the diagnosis of acute appendicitis. J Pak Med Stud 2011; 1 : 32-37.

10 Xharra S, Gashi-Luci L, Xharra K, et al. Correlation of serum C-reactive protein, white blood count and neutrophil percentage with histopathology findings in acute appendicitis. World J Emerg Surg 2012; 7:27.

11 Yesiltas M, Karakas DO, Gokcek B, Hot S, Egin S. Can Alvarado and appendicitis inflammatory response scores evaluate the severity of acute appendicitis? Ulus Travma Acil Cerrahi Derg 2018; 24 : 557-562.

12 Chong C F, Thien A, Mackie AJ, et al. Comparison of RIPASA and Alvarado scores for the diagnosis of acute appendicitis. Singapore Med J 2011;52 : 340-345.

13 Sanjive JG, Ramaiah RH. Comparison of RIPASA and Alvarado scoring in the diagnosis of acute appendicitis and validation of RIPASA scoring. Int Surg J 2019;6 : 935-939.

14 El-Shamy A. Prognostication of pediatric appendicitis with three scoring system. Life Sci J 2017;14 : $17-24$.

15 Nanjundaiah N, Mohammed A, Shanbhag V, Ashfaque K, Priya SA. A comparative study of RIPASA score and Alvarado score in the diagnosis of acute appendicitis. J Clin Diagn Res 2014;8 : 03-05.

16 Karami MY, Niakan H, Zadebagheri N, Mardani P, Shayan Z, Deilami I. Which one is better? comparison of the acute inflammatory response, Raja Isteri Pengiran Anak Saleha Appendicitis and Alvarado scoring systems. Ann Coloproctol 2017; $33: 227-231$.

17 Noor S, Wahab A, Afridi G, Ullah K. Comparıng RIPASA score and alvarado score in an accurate diagnosis of acute appendicitıs. J Ayub Med Coll Abbottabad 2020;32 : 38-41.

18 Dezfuli SAT, Yazdani R, Khorasani M, Hosseinikhah SA. Comparison between the specificity and sensitivity of the RIPASA and Alvarado Scoring systems in the diagnosis of acute appendicitis among patients with complaints of right iliac fossa. AIMS Public Health 2020 7:1-9.

19 Díaz-Barrientos CZ, Aquino-González A, Heredia-Montaño M, Navarro-Tovar F, Pineda-Espinosa MA, Espinosa de Santillana IA. The RIPASA score for the diagnosis of acute appendicitis: A comparison with the modified Alvarado score. Rev Gastroenterol Mex 2018; 83 : 112-116.

20 Frountzas M , Stergios K, Kopsini D, Schizas D, Kontzoglou K, Toutouzas K. Alvarado or RIPASA score for diagnosis of acute appendicitis? a meta-analysis of randomized trials. Int J Surg 2018; 56 : 307-314.

21 Arroyo-Rangel C, Limón IO, Vera ÁG, Guardiola PM, Sánchez-Valdivieso EA. Sensitivity, specificity and reliability of the RIPASA score for diagnosis of acute appendicitis in relation to the Alvarado Score. Cir Esp 2018; $96: 149-154$.

22 Golden SK, Harringa JB, Pickhardt PI, et al. Prospective evaluation of the ability of clinical scoring systems and physician-determined likelihood of appendicitis to obviate the need for computed tomography. Emerg Med J 2016; 33 : 458-464.

\section{Hosted file}


Table 1.pdf available at https://authorea.com/users/374596/articles/492081-alvarado-versusraja-isteri-pengiran-anak-saleha-appendicitis-scores-for-diagnosis-acute-appendicitisin-children

\section{Hosted file}

Table 2.pdf available at https://authorea.com/users/374596/articles/492081-alvarado-versusraja-isteri-pengiran-anak-saleha-appendicitis-scores-for-diagnosis-acute-appendicitisin-children

\section{Hosted file}

Table 3.pdf available at https://authorea.com/users/374596/articles/492081-alvarado-versusraja-isteri-pengiran-anak-saleha-appendicitis-scores-for-diagnosis-acute-appendicitisin-children

\section{Hosted file}

Table 4.pdf available at https://authorea.com/users/374596/articles/492081-alvarado-versusraja-isteri-pengiran-anak-saleha-appendicitis-scores-for-diagnosis-acute-appendicitisin-children

\section{Hosted file}

Table 5.pdf available at https://authorea.com/users/374596/articles/492081-alvarado-versusraja-isteri-pengiran-anak-saleha-appendicitis-scores-for-diagnosis-acute-appendicitisin-children 